\title{
Comparison of Tongue and Lip Trills with Phonation of the Sustained Vowel $/ \varepsilon$ / Regarding the Periodicity of the Electroglottographic Waveform and the Amplitude of the Electroglottographic Signal
}

\author{
Gislaine Ferro Cordeiro ${ }^{1^{*}}$, Arlindo Neto Montagnoli², Maysa Tibério Ubrig3, \\ Marcia Helena Moreira Menezes ${ }^{4}$, Domingos Hiroshi Tsuji ${ }^{3}$ \\ ${ }^{1}$ ENT Department, School of Medicine, University of Sao Paulo, São Paulo, Brazil \\ ${ }^{2}$ Electrical Engineering Department, Federal University of Sao Carlos, São Paulo, Brazil \\ ${ }^{3}$ School of Medicine, University of Sao Paulo, São Paulo, Brazil \\ ${ }^{4}$ Speech and Hearing Department, Guarulho University, Guarulhos, Brazil \\ Email: "gislainecordeiro@gmail.com
}

Received 5 February 2014; accepted 27 December 2015; published 30 December 2015

Copyright (C) 2015 by authors and Scientific Research Publishing Inc.

This work is licensed under the Creative Commons Attribution International License (CC BY). http://creativecommons.org/licenses/by/4.0/

(c) (i) Open Access

\section{Abstract}

Aim: The aim is to compare the vocal fold vibration seen during lip and tongue trills with that seen during phonation of the sustained vowel $/ \varepsilon /$, in terms of the periodicity of the EGG waveform and the amplitude of the EGG signal, in professional voice users. Study design: This was a quasi-experimental study. Methods: We used electroglottography (EGG) to compare the vocal fold vibration seen during tongue and lip trills with that seen during phonation of the sustained vowel $/ \varepsilon /$, in terms of the EGG waveform periodicity and signal amplitude, in 10 classically trained, professional singers. The participants produced the sustained vowel $/ \varepsilon /$ and performed tongue and lip trills at the same frequency and intensity. The periodicity of the waveform and the amplitude of the signal were visually analyzed by three blinded, experienced readers. To confirm the visual analysis results, we measured the jitter and shimmer of the signal and the frequency of variation in vocal fold vibration during the trill exercises. Results: The EGG waveform was classified as periodic for the sustained vowel phonation task and as quasi-periodic for the trill exercises, the vibration pattern repeating at approximately $24 \mathrm{~Hz}$. Conclusion: The vibration of the vocal folds was modified according to the supraglottic movement in trills exercises.

\footnotetext{
"Corresponding author.
} 


\section{Keywords}

\section{Vibration, Electroglottography, Amplitude, Waveform, Vocal Folds}

\section{Introduction}

Articulators are a group of structures that work collectively to maintain the resistance of the vocal tract during phonation. The speech system is highly capable of adjusting the articulation and breath to maintain the aerodynamics during the production of consonants, especially plosives [1] [2].

One of the purposes of using facilitating sounds during vocal training is to intensify the interaction between the larynx and the supraglottic vocal tract, which may increase vocal intensity, efficiency, and economy [3].

Some changes can be seen in vocal tract impedance when the vocal tract is partially occluded, or when the tube is extended, for example with straws. The longer the tube extension or the occlusion is, the higher the impedance is. Exercises that cause the vocal tract to completely occlude—for instance, a long $/ b /$ - cause high impedance, and the voice is produced for a short period. Even though exercises for voiced fricatives such as bilabial [b:] also increase intraoral pressure and, consequently, the impedance; their load is a bit lower because the occlusion is partial [4].

Lip and tongue trills, humming, nasal consonants, exercises using tubes and straws, and voiced fricatives are considered as exercises of semi-occluded vocal tract because they offer intraoral pressure, promoting sourcefilter interaction [3].

The difference between voiced trills of lips and tongue and other semi-occluded vocal tract exercises is that the former promotes the occlusion and non-occlusion of the point of articulation (without reducing muscle tonus), generating some difference in supraglottic oscillatory pressure. During these exercises, the air flow and supraglottic pressure must be correct to cause both the articulation and the vocal folds to vibrate at the same time, overloading the laryngeal adductor muscles [5]. The frequency of the articulatory vibration in this exercise ranges from 25 to $35 \mathrm{~Hz}$ [6].

These exercises are widely spread among speech-language pathologists, vocal coaches and other voice instructors, and they are indicated for the treatment of hyperkinetic and hypokinetic pathologies [7]-[10], as well as for voice warm-up [10]-[14]. Their post-treatment efficiency has already been proven [15].

In normal voices, the voiced trill of the tongue promotes higher amplitude in the vibration of vocal folds, reduction in the glottic gaps [16], and improvements in the evaluation of acoustic and auditory perception, reducing the shimmer, increasing the harmonics-to-noise ratio and the amplitude of harmonics, and reducing the noise [17] [18].

There is little research on the effects of voiced trill exercises for the lips and tongue during their execution. Such research shows that, when the tongue vibrates, the entire laryngeal framework vibrates, and the anteroposterior pharynx is constricted [19]. Furthermore, when the tongue or lips vibrate, the amplitude of the vibration of the mucosa in the vocal folds is higher than that of the sustained vowel [20], and the contact coefficient decreases [6] [21] and varies [22].

Through a high-speed video exam, the execution of voiced vibration of the aryepiglottic folds is found to interact with the vibration of the vocal folds and to cause alternation in the vibration model of the vocal folds [23].

Considering the mathematical models that study the interactions between source and filter, the variations in the contact coefficient, and the difficulty in synchronizing the stroboscopic light during the execution of voiced exercises for the tongue and lips can be supposed that there is some interference in the oscillatory vibration of vocal folds, according to the variation in the supraglottic pressure caused by the oscillation of the point of articulation in voiced trills of lips and tongue. However, there are no studies that detail the vibration of vocal folds during the execution of these exercises, or that prove this theory in human beings.

Detailing the vibration of vocal folds during the execution of exercises may support the clinical rationale. Theoretical confirmation supports prescription and gives credibility to the treatment argument. Thus, a deeper understanding of the mechanism of vocal fold vibration during vocal exercises [including tongue and lip trills) can assist speech-language pathologists in prescribing the exercises. 
Concomitant use of EGG and high-speed video recording constitutes the best method for studying the physiology of glottal vibration. However, high-speed videography with a fiberoptic laryngoscope is currently unfeasible because the nasolaryngoscopes currently available do not provide enough illumination to allow adequate visualization of the vocal folds. In which case, the EGG is the best procedure.

Our objective is to compare the vocal fold vibration seen during lip and tongue trills with that seen during phonation of the sustained vowel $/ \varepsilon /$, in terms of the periodicity of the EGG waveform and the amplitude of the EGG signal, in professional voice users.

\section{Methods}

The study was approved by the Research Ethics Committee of the Hospital das Clínicas da Faculdade de Medicina da Universidade de São Paulo (HCFMUSP, University of São Paulo School of Medicine Hospital das Clínicas; Protocol No. 907/06, February 14, 2007), located in the city of São Paulo, Brazil. All of the study participants gave written informed consent.

\subsection{Study Sample}

In the present study, we evaluated fourteen classically trained, professional singers (seven males and seven females). We applied the following criteria:

- Inclusion criteria - being a healthy, classically trained, professional singer; having been singing professionally for at least three years; having laryngeal control; having mastered the techniques for performing lip and tongue trills; and presenting with no vocal fold lesions.

- Exclusion criteria - presenting with singing or speaking voice complaints; presenting with incomplete glottal closure; not tolerating examination of the larynx; and producing grade 3 or 4 EGG waveforms, as graded by two speech-language pathologists with experience in EGG and one electrical engineer, in accordance with the grading system proposed by Vieira [24].

Four individuals (two males and two females) were excluded. Of the two males, one was excluded because he could not tolerate the examination of the larynx and one was excluded because he had been a professional singer for less than 3 years ( 1 year and 6 months). Of the two females, one was excluded because she could not tolerate the examination of the larynx and one was excluded because she presented with speaking voice complaints.

Therefore, we evaluated ten singers. We chose to evaluate classically trained singers because we believe that this is the only way to isolate the production of exercises, since singers have greater control of the vocal tract and are therefore able to perform trill exercises employing only those structures that are required for an ideal performance.

\subsection{Preparation for Data Collection}

Prior to data collection, we measured the vocal range of each participant. Vocal range is the distance between the lowest and the highest note that an individual can produce, excluding the vocal fry register and including the falsetto [25]. After having measured the vocal range of the participants, we selected the 5th whole step above the lowest possible note that each individual was able to produce [26]. The participants were then asked to produce, in that note and with their larynx in a low position, the sustained vowel $/ \varepsilon /$, lip trills, and tongue trills, as well as the voiced fricatives $/ v /, \mid z /$, and $/ 3 /$, at the highest and lowest possible intensities of which they were capable. As shown in Table 1, we chose the lowest of the highest intensities and the highest of the lowest intensities, in order to standardize the intensities during the performance of the exercises.

The voiced fricatives were used because the highest of the lowest intensities is commonly achieved during the phonation of the sounds evaluated. Therefore, the participants were able to perform the tests comfortably without

Table 1. Highest and lowest intensities achieved by individual 1 during sustained phonations. The values chosen for data collection were $52 \mathrm{~dB}$ for the lowest intensity and $68 \mathrm{~dB}$ for the highest intensity.

\begin{tabular}{|c|c|c|c|c|c|c|}
\hline Intensity & Vowel / $\varepsilon /$ & Lip trill & Tongue trill & $/ \mathbf{v} /$ & $\mid \mathbf{z} /$ & $/ 3 /$ \\
\hline Lowest (dB) & 51 & $\underline{52}$ & 52 & $<50$ & $<50$ & 51 \\
\hline Highest (dB) & 85 & 70 & 71 & $\underline{68}$ & 70 & 71 \\
\hline
\end{tabular}


experiencing vocal fatigue or aperiodicity due to the use of threshold-range phonation during the performance of trill exercises. In order to measure vocal range, as well as to determine and maintain the selected note. Intensity was measured with a sound pressure level meter (model 33-2055, RadioShack Corporation, Fort Worth, TX, USA) placed $30 \mathrm{~cm}$ from the corner of the mouth of the singer. After the abovementioned procedure, each singer practiced producing the sounds at the requested note and at the intensity selected (maximum variation, $2 \mathrm{~dB}$ ), with minimal effort and maintaining the larynx in the same (low) position, for $\geq 10$ seconds.

The practice session was conducted with the aid of a speech-language pathologist specializing in voice, who instructed the singers during a meeting held before data collection. On the day of data collection, previously agreed upon by the singers and the investigators, the phonation tasks were performed again before each test. In order to avoid the problems of participants presenting with the flu, sleep deprivation, vocal fatigue, or voice disorders on the day of data collection, the participants were informed of the objectives and conditions of the present study before the first evaluation was scheduled.

\subsection{Data Collection and Analysis}

\subsubsection{Videolaryngostroboscopy}

All of the singers underwent videolaryngostroboscopy in order to exclude laryngeal diseases. The videolaryngostroboscopy was conducted by an experienced otolaryngologist of the HCFMUSP Clinical Otolaryngology Division Voice Group Outpatient Clinic.

\subsubsection{EGG}

The individuals underwent EGG at the Centro de Especialização em Fonoaudiologia Clínica (CEFAC, Center for Clinical Audiology and Speech-Language Pathology), in the city of São Paulo. For the tests, the individuals were asked to produce the sustained vowel $/ \varepsilon /$ and perform tongue and lip trills for as long as possible at the same frequency and intensities as those used in the training sessions (5th whole step above the lowest note in their range and the lowest of the highest/highest of the lowest, respectively). The singers controlled the frequency, using the same keyboard that was used in the training sessions. Intensity was monitored by a speechlanguage pathologist in the examination room, with the same sound pressure level meter that was used in the training sessions, which was again positioned at $30 \mathrm{~cm}$ from the corner of the mouth of the singer. Moreover, they had to try and keep their larynx fixed at a lower position to reduce the effects of larynx movements in the results of EGG waveforms.

We made intraindividual comparisons. Low-intensity phonations were compared only with one another, as were high-intensity phonations.

In order to measure the EGG signal, we referred the individuals to a sound-treated booth in a quiet room in the CEFAC Voice and Speech Laboratory. We used an EG2-Standard electroglottograph (Glottal Enterprises, Syracuse, NY, USA). The electroglottograph was connected to a computer audio interface (BCA2000; MUSIC Group Services EU GmbH, Willich, Germany), which was in turn connected to a computer.

The individuals were asked to remove any metal objects that they might be wearing in the head and neck region and to sit upright in a chair equipped with a headrest. To ensure the safety of the individuals, we placed a rubber mat under the chair. The neck region was cleaned with dry paper towel. We applied a thin layer of hypoallergenic electrically conductive gel (SPECTRA $360^{\circledR}$; Parker Laboratories, Inc., Fairfield, NJ, USA) to the electrodes, which were placed over each ala of the thyroid cartilage and secured with a Velcro strap around the neck. To ensure that the electrodes were positioned correctly, we asked the participants to produce the sustained vowel and perform tongue trills. We then observed whether the green light-emitting diodes (LEDs) of the top LED array (Electrode Placement/Laryngeal Movement) on the front panel of the electroglottograph were lit. To confirm that there was signal, we observed whether the green LEDs of the bottom LED array (signal) were lit. To record the signal, we selected the Vocal Fold Contact Area signal option, and the high or low gain option of the device was determined by monitoring the signal with the LED signal indicator. The signal was saved as a .wav file and edited with the audio editing suite Sound Forge, version 7.0 (Sony Creative Software, Inc., Middleton, WI, USA), at a sampling frequency of 22,050 Hz and a resolution of 16 bits. The EGG waveforms were submitted to high band-pass filtering with the program delay [24], designed by Maurílio Nunes Vieira, an engineer at the Federal University of Minas Gerais, located in the city of Belo Horizonte, Brazil. The high-pass filter is used to isolate the functioning of the glottis. However, to make sure that the signals obtained result from the 
electrical sign of glottic movements, without any interference of articulatory movements, a test was performed, in which the subjects, simultaneously recorded in audio and in EGG, had to trill the tongue and lips, alternating between voicing and not voicing in the same exhalation (Figure 1).

The waveforms were initially graded by two speech-language pathologists with experience in EGG and one electrical engineer, in accordance with the grading system proposed by Vieira [24]:

- Grade 1, free of evident noise;

- Grade 2, increased intensity but with visible noise;

- Grade 3, irregular excitation;

- Grade 4, impossible to analyze numerically.

Subsequently, the samples were analyzed by three speech-language pathologists with experience in acoustic analysis, who analyzed the periodicity of the EGG waveforms and the amplitude of the EGG signal. To that end, the images on Sound Forge (Sony Creative Software, Inc.) were captured by pressing the Print Screen key on the computer keyboard. For each phonation task, we selected the most stable snippets of the EGG waveform, at zoom ratios of 1:2 and 1:8 for male singers and 1:4 and 1:16 for female singers (Figure 2).

The images were edited with the program Microsoft Paint and pasted onto a Microsoft Word document. As can be seen in Figure 1 and Figure 2, the images obtained at higher zoom ratios (1:2 or 1:4) were pasted immediately below those obtained at lower zoom ratios (1:8 or 1:16), being subsequently analyzed. The order in which the study participants performed the phonation tasks was determined by random sampling, so that the raters

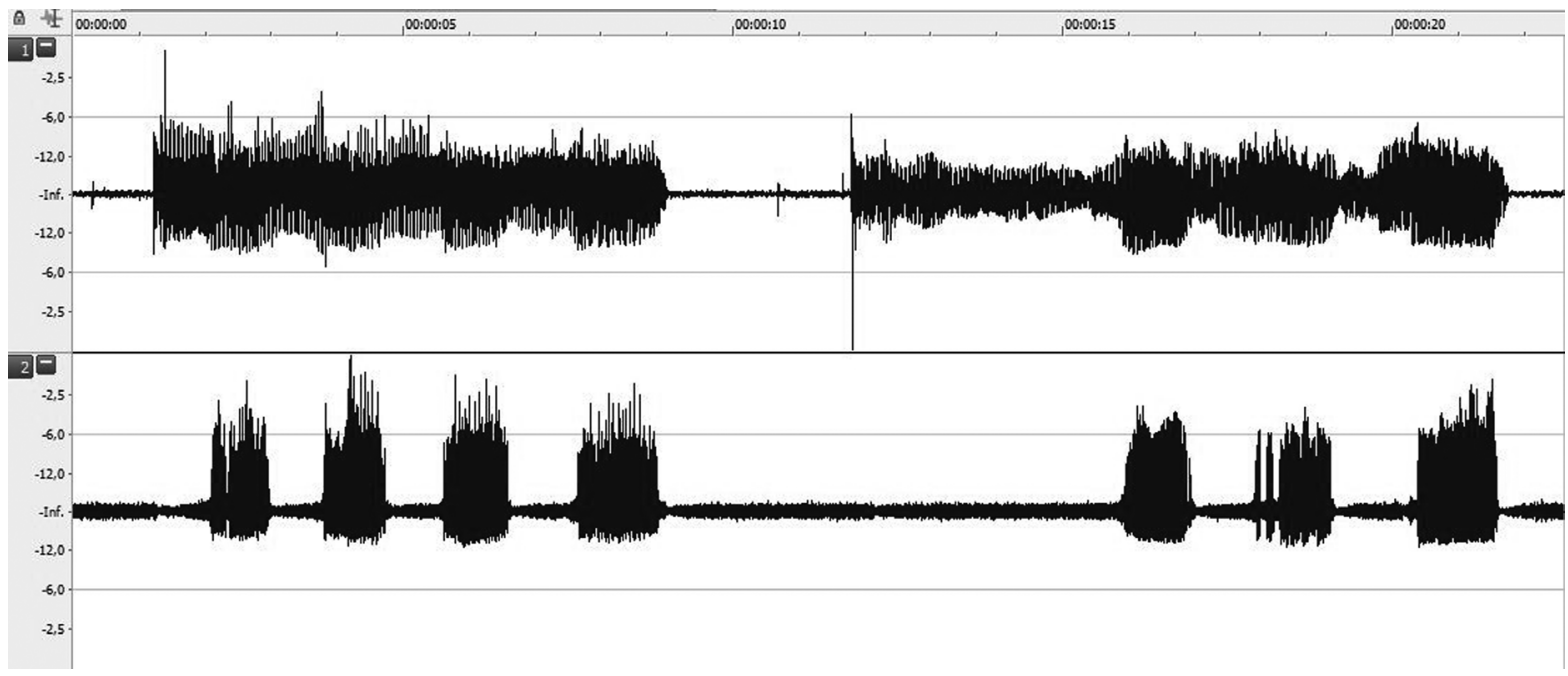

Figure 1. Simultaneous recorded in audio and in EGG, had to trill the tongue and lips, alternating between voicing and not voicing in the same exhalation.
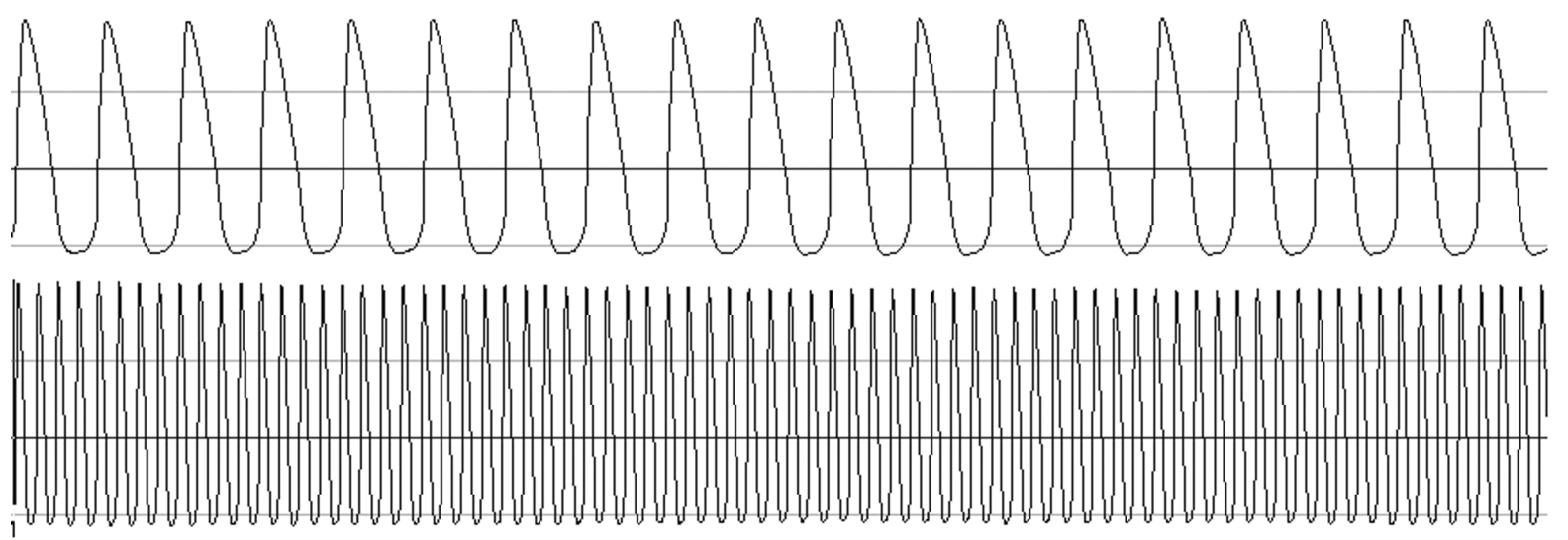

Figure 2. Electroglottographic waveforms for the sustained vowel $/ \varepsilon /$ produced at high intensity by a male individual, at zoom ratios of $1: 2$ and $1: 8$. 
were blinded to the phonation task being analyzed. The EGG waveforms obtained during phonation tasks performed at high intensity were analyzed separately from those obtained during phonation tasks performed at low intensity. In order to determine the reliability of the answers provided by the raters, $10 \%$ of all images were analyzed twice. The raters received a handout containing 33 images for waveform periodicity at each intensity and 33 images for waveform amplitude at each intensity. Therefore, each rater analyzed a total of 132 images.

\subsubsection{Visual Analysis of the EGG Waveform and Signal}

Before performing visual analysis of the EGG waveform and signal, the raters participated in a training session, in which EGG waveforms produced by 3 individuals who had been excluded from the present study were used. Visual analysis of the EGG waveform and signal took into account periodicity and amplitude oscillation, respectively.

Periodicity of the EGG waveform

On the basis of periodicity, the EGG waveform was classified as follows:

- Periodic waveform-a waveform that repeats itself at regular intervals (Figure 3);

- Quasi-periodic waveform-a waveform that does not repeat itself in the shorter term but does so in the longer term (Figure 4);

- Aperiodic waveform-a waveform that has no repeating pattern (Figure 5).

Amplitude oscillation of the EGG signal

On the basis of amplitude oscillation, the EGG signal was classified as follows:

- Non-oscillating - the amplitude of the EGG signal does not oscillate or have a repeating pattern over time (Figure 6);

- Oscillating — the amplitude of the EGG signal oscillates and has a repeating pattern over time (Figure 7).

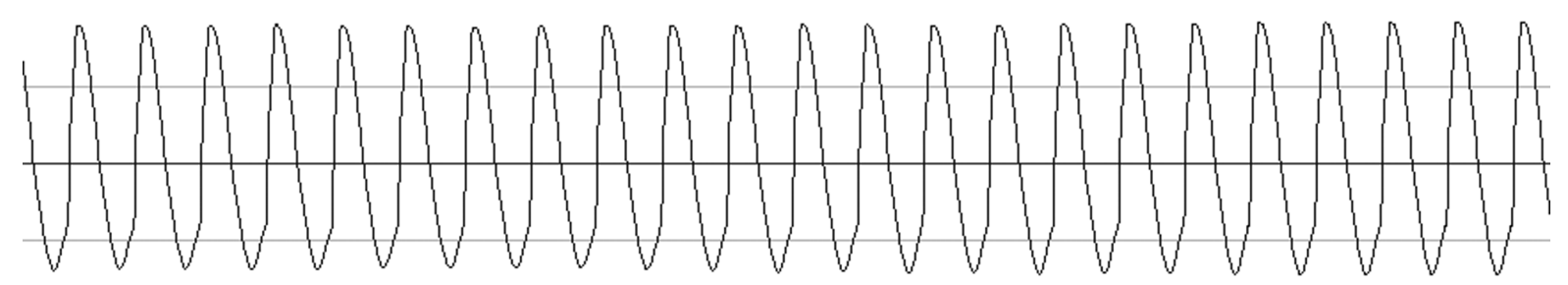

Figure 3. Periodic electroglottographic waveform.

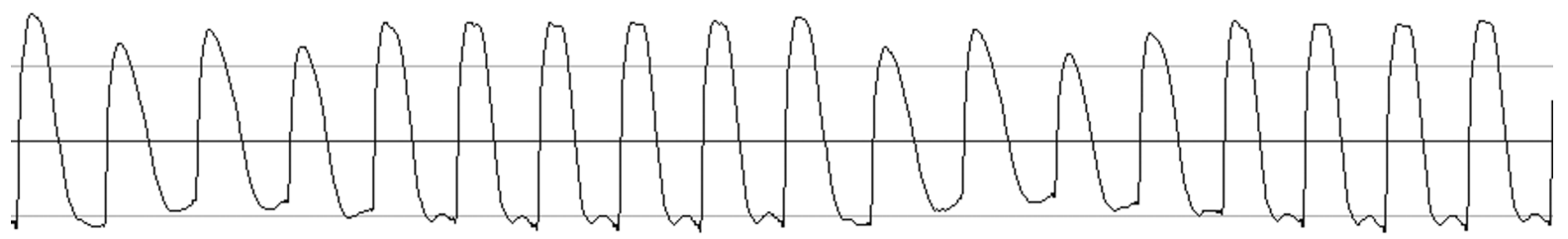

Figure 4. Quasi-periodic electroglottographic waveform.

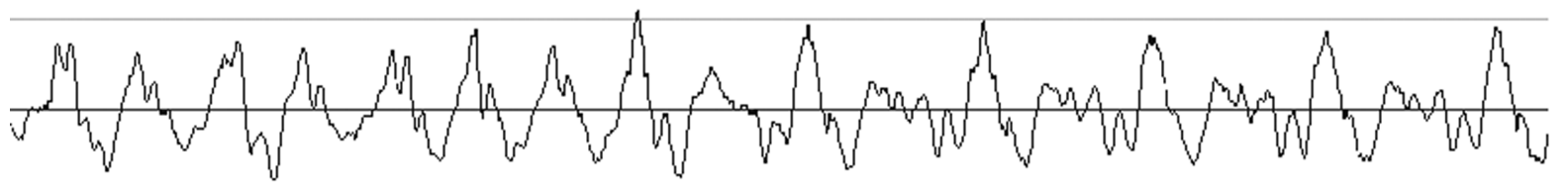

Figure 5. Aperiodic electroglottographic waveform.

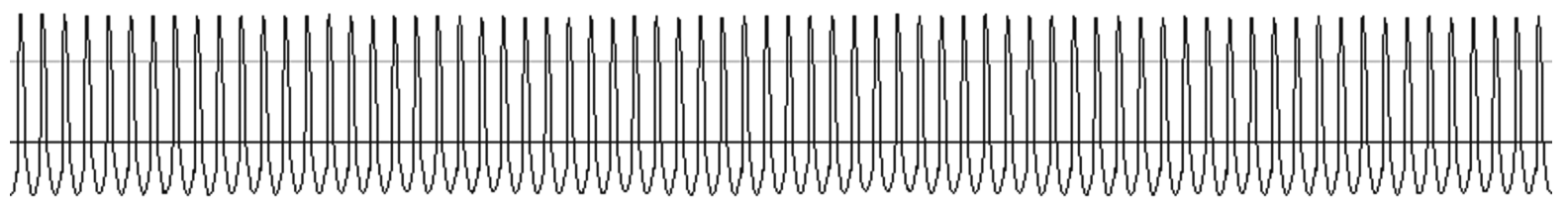

Figure 6. Non-oscillating electroglottographic signal: the amplitude of the signal does not oscillate. 


\subsubsection{Measurements of the EGG Waveforms}

The EGG waveforms were measured with an automatic system developed by Vieira [25] and made available for use in the present study. The automatic measurements were removed from the middle of the sample for, approximately, 3 seconds. The measurements taken were jitter (percentage of frequency perturbation) and shimmer RMS (percentage of amplitude perturbation), the values of which corresponded to the visual analyses of the EGG waveform and signal by the three raters.

Additionally, the amplitude value of the peaks of $25 \mathrm{Lx}$ waveforms from the exercises of voiced trills of lips and tongue were measured. From these values, the highest value of the amplitude cycle was identified, and the number of cycles between each waveform was counted based on the identified waves, as shown in Figure 8. After that, the relation between the fundamental frequency and the number of cycles was established in order to measure the frequency rate of the Lx waveform oscillation during the execution of exercises of voiced trills of tongue and lips. The average and standard deviation of these values were obtained.

\subsection{Statistical Analysis}

In order to analyze the results, we adopted a level of significance of $5 \%(P=0.05)$ for all statistical tests. The IBM SPSS Statistics software package, version 17.0 (IBM Corporation, Armonk, NY, USA), was used for the analysis.

We used Friedman's test in order to determine the differences among phonation of the sustained vowel $/ \varepsilon /$, lip trills, and tongue trills in terms of the automatic measurements taken. For the cases in which the difference was statistically significant, we used the Wilcoxon signed rank test in order to identify the types that differed.

Cronbach's alpha coefficient was used in order to determine the reliability (internal consistency) of the data provided by the raters. Cronbach's alpha coefficient values were high $(<0.001$ to 0.006 , range, $0.7-1.0)$, indicating high inter-rater reliability. Therefore, one of the three raters (the first) was randomly selected to be the data provider. Friedman's test was used for data analysis.

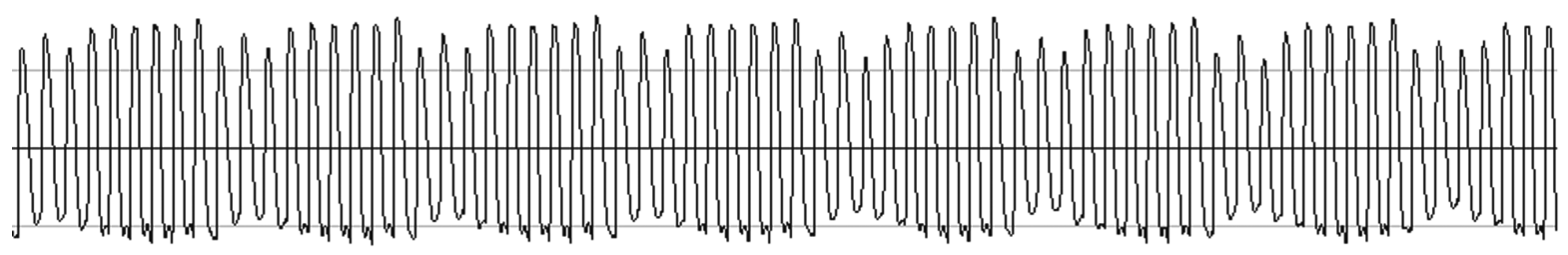

Figure 7. Oscillating electroglottographic signal: the amplitude of the signal oscillates between large and small.

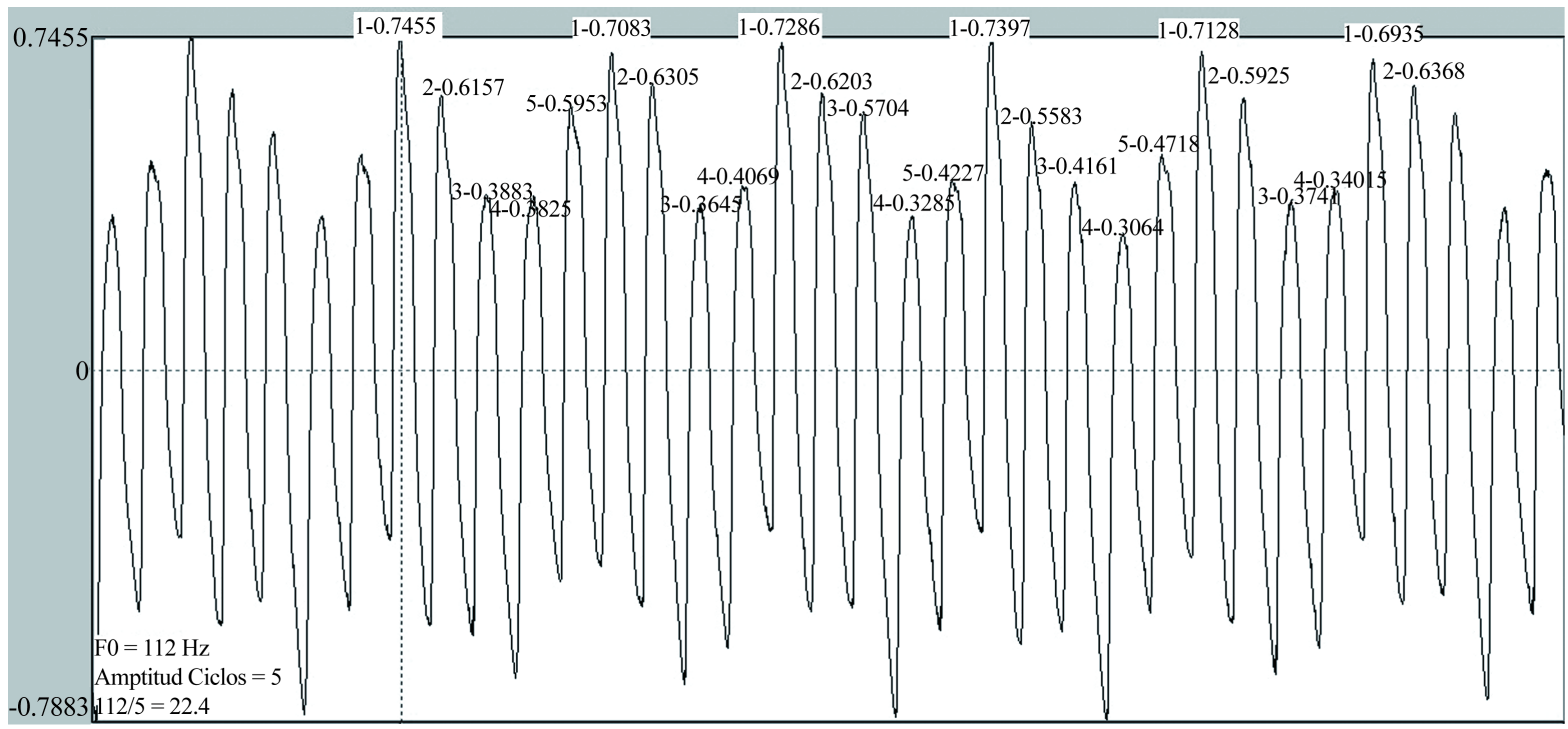

Figure 8. Rate of the Lx waveform oscilation. The measured is the relation between the fundamental frequency and the number of cycles between largest values of amplitude. 


\section{Results}

\section{Visual-Perceptual Analysis}

Regardless of the intensity at which they were performed, tongue trills and lip trills were found to be similar in terms of the periodicity of the EGG waveform, the amplitude of the EGG signal and the automatic measurements of jitter and shimmer, having differed, however, from the sustained vowel $/ \varepsilon /$.

\section{Periodicity and amplitude of the EGG waveform:}

A periodic EGG waveform for the sustained vowel phonation task (Figure 4) and a quasi-periodic EGG waveform for lip and tongue trills (Figure 5) $-p$ value of equal to 0.001 . The amplitude of the EGG signal was classified as non-oscillating for the sustained vowel phonation task (Figure 7) and as oscillating for lip and tongue trills (Figure 8 ) - $p$ value of equal 0.001 .

Measurements of the EGG waveforms:

The jitter and shimmer RMS values were higher for tongue and lip trills performed at high and low intensities than for the sustained vowel $/ \varepsilon /$ produced at high and low intensities.

In high intensity, jitter values were $0.23 \%, 2.94 \%$, and $3.46 \%$ for the sustained vowel, voiced lip trill, and voiced tongue trill. The standard deviations were $0.05,1.20$, and 0.75 , respectively. In low intensity, jitter values were $0.78 \%$, $3.46 \%$, and $3.52 \%$ with standard deviations of $0.75,0.91$, and 1.00 for the sustained vowel, voiced lip trill, and voiced tongue trill, respectively.

In high intensity, shimmer values were $0.65 \%, 12.07 \%$, and $6.55 \%$ with standard deviations of $0.46,2.76$, and 2.77 for the sustained vowel, voiced lip trill, and voiced tongue trill, respectively. In low intensity, shimmer values were $3.72 \%, 18.23 \%$, and $19.31 \%$ for the sustained vowel, voiced lip trill, and voiced tongue trill. The standard deviations were $3.57,8.79$, and 12.46 , respectively.

In high and low intensities, both for jitter and shimmer, the exercises of voiced lip and tongue trills were similar to each other and different from the sustained vowel, according to Wilcoxon signed rank test.

The average of the frequency rate of the amplitude oscillation in high intensities was $22.4 \mathrm{~Hz}$ for voiced lip trills and $25.2 \mathrm{~Hz}$ for voiced tongue trills. The standard deviations were 3.2 and 2.3, respectively. In low intensities, the average for voiced lip trills was $23.7 \mathrm{~Hz}$, and the standard deviation was 4.7; and the values for voiced tongue trills were $25.7 \mathrm{~Hz}$, and 5.2, respectively.

\section{Discussion}

In the present study, tongue trills and lip trills performed by professional singers at high and low intensities were similar in terms of the periodicity of the EGG waveform and the amplitude of the EGG signal, having differed, however, from the sustained vowel $/ \varepsilon /$ produced at high and low intensities. Although the present study was an exploratory study including only ten individuals, it provided strong evidence for the differences between trill exercises and phonation of the sustained vowel $/ \varepsilon /$ in terms of the periodicity of the EGG waveform and the amplitude of the EGG signal. Therefore, we believe that the results would not have been dramatically different had we studied a larger sample of individuals.

The difference between trill exercises and phonation of the sustained vowel $/ \varepsilon /$ was quite clear, given the high level of inter-rater agreement, Cronbach's alpha coefficient being approximately 0.9 for most analyses. As can be seen in Figure 3 and Figure 4, the EGG waveform was classified as periodic for the sustained vowel phonation task and as quasi-periodic the trill exercises. The results of the visual analysis of the EGG waveform were consistent with the automatic measurements of jitter, which were found to be higher during the trill exercises than during the sustained vowel phonation task.

Cordeiro et al found that the closed quotient varies widely during trill exercises [20] [22]. In addition, the authors reported difficulty in synchronizing the flashes of light from the stroboscope with the vocal fold vibration during lip and tongue trills. The results of the present study corroborate the hypothesis that the difficulty in synchronizing the flashes of light from the stroboscope with the vocal fold vibration during lip and tongue trills is due to signal aperiodicity [20].

For the performance of lip trills and tongue trills, pulmonary airflow has to increase in order to maintain vocal fold vibration and lip or tongue vibration [1] [6] [26]-[28]. Increased airflow can lead to aperiodicity of vocal fold vibration [29].

If we chose to explain phonation on the basis of the Bernoulli equation, we would have to assume that glottal 
flow is unidirectional, stable, and predictable [30]. However, this would not fully explain our results. Therefore, we chose to explain the mechanism of tongue and lip trills on the basis of the theories of glottal vibration and fluid mechanics, both of which have been investigated more recently.

Some authors have reported that the sound produced by the larynx is nonlinear [29] [31]-[34], which means that the phonation cycle loses its regularity when subglottic pressure is higher than the optimal phonation pressure [29]. The non-periodicity of speech cycles may be caused by the nonlinearity of the system assigned to the Coanda effect [30] [35]-[37], as well as to the presence of vortices in the glottic region [38]-[40] and in the supraglottic region [33] [38] [40]-[42]. Increased airflow increases these effects [36] [41]-[43].

The Coanda effect is the tendency of a fluid (or air, in the case of glottal vibration) to adhere to a solid surface (principally a convex surface) and change its course in accordance with the shape of the surface [36] [37] [44]-[46]. By the Coanda effect, the air layers that are farthest from the surface travel faster than do those that are closest to the surface [45]. The fluid exerts a force on the surface. Therefore, we believe that, by the Coanda effect, the force that airflow exerts on the vocal folds when they are closed leads to vocal fold separation [47], and the negative pressure caused by the higher velocity of the molecules adjacent to those attached to the surface can explain the closing phase.

Vorticity is the tendency of a fluid to spin as a result of the tendency of a system to balance the differences in pressure among neighboring areas. Vortices typically occur when the speed of the airflow exceeds a certain limit, which depends on the nature and temperature of the fluid [43]. Vortices are characterized by negative static pressures [29], and the presence of vortices in the vocal folds exerts an additional force on glottic closure [29] [32], as well as producing an unsteady flow at the exit of the glottis [38] [39]. Vortices occur primarily in the divergent phase of the glottal cycle [39] [40] [42] [48].

In our study, the EGG waveform for lip and tongue trills was quasi-periodic, supporting the idea that there is variation in glottal airflow. This variation can lead to variations in the intensity of the effects described above, resulting in a quasi-periodic waveform. In addition, the amplitude of the EGG signal oscillated during the performance of trill exercises (Figure 7), which did not occur during the sustained vowel phonation task. We found significant differences between the sustained vowel phonation task performed at low intensity and the trill exercises performed at low intensity $(P=0.007)$, as well as between the sustained vowel phonation task performed at high intensity and the trill exercises performed at high intensity $(P=0.001)$, in terms of shimmer (as measured automatically), shimmer values therefore corroborating the results of the visual analysis. The Wilcoxon signed rank test revealed significant differences between lip trills and phonation of the sustained vowel $/ \varepsilon /$, as well as between tongue trills and phonation of the sustained vowel $/ \varepsilon /$, without any such differences between lip trills and tongue trills.

Signal amplitude and, consequently, shimmer as assessed by EGG are different from signal amplitude and shimmer as assessed by acoustic analysis [49]. Signal amplitude as assessed by acoustic analysis is related to vocal intensity. Signal amplitude as assessed by EGG is related to the electrical conductance of the larynx, larger EGG signal amplitude translating to greater vocal fold contact [24]. This variable is highly sensitive to even the slightest changes in the amplitude of the signal [50].

Because the amplitude of vocal fold vibration oscillates, we can assume that the vocal fold contact area varies during tongue and lip trills. In the present study, the average of the frequency rate of the amplitude oscillation of the EGG signal was $22.4 \mathrm{~Hz}$ during lip trills performed at high intensity, $25.2 \mathrm{~Hz}$ during tongue trills performed at high intensity, $23.7 \mathrm{~Hz}$ during lip trills performed at low intensity, and $25.2 \mathrm{~Hz}$ during tongue trills performed at low intensity. The abovementioned values for tongue trills are similar to the 25 - $35 \mathrm{~Hz}$ reported in the literature [6].

For tongue trills and lip trills to occur, the anterior part of the vocal tract must be occluded by the tongue or the lips. Intraoral pressure increases and becomes greater than the force that maintains the anterior part of the vocal tract closed; therefore, the anterior part of the vocal tract opens and is "sucked out" by the speed of the airflow [6]. Therefore, supraglottic pressure oscillates as the tongue or the lips vibrate. We therefore believe that the presence of vortices in the supraglottic region is increased when intraoral pressure is high and decreased when intraoral pressure is low. If we assume that the presence of vortices increases vocal fold contact during closure [41], then variations in the presence of vortices lead to variations in vocal fold contact. This explains the variation in the amplitude of the EGG signal and the increase in shimmer in the present study.

The theory of source-filter interaction [51] [52] states that the acoustic pressure in the vocal tract changes the phonation threshold pressure and interferes with vocal fold vibration. Following this line of reasoning, Titze [3] 
reported that the objective of voice training is to promote the interaction between the source and the filter and therefore increase vocal intensity, efficiency, and economy. According to the author, lip trills and tongue trills are among the SOVTEs. Because SOVTEs promote a mechanical interaction between the source and the filter [51] [52], they change vocal fold impedance and therefore inhibit vocal fold vibration [4]. Our results confirm the theory that is widely studied in mathematical models, in human beings. Nevertheless, we can suppose that the effects caused by exercises that promote the constant partial obstruction of the vocal tract, such as voiced fricatives, are different from those that promote oscillatory obstruction, being the therapeutic indications also different.

Furthermore, the strength and speed applied onto the point of articulation during the execution of vibrations may alter the effect of vocal fold vibration. Therefore, clinicians must pay close attention to the form of execution in accordance with their objectives. Other studies may be carried out to detail these factors, so that the indication is even more precise and bring therapeutic results in even less time.

Pinho [25] [53] supposes that trill exercises massage the vocal folds. We believe this hypothesis because these exercises cause major changes in vocal fold vibration. During trill exercises, articulatory vibration is added to the glottal vibration. In our study, the values of the frequency of amplitude oscillation of Lx waveforms are similar to the values of the articulatory oscillation in lip trills described in the literature. Given that intraglottal pressure distribution determines the effect of the force on the vocal fold wall [47], we presume that intraglottal pressure oscillation can be compared to a vocal fold massage. A body massage improves the flexibility of adjacent tissues [54] and increases blood flow in the region [14] [53], thus increasing the supply of oxygen, nutrients, and hormones [55]; removing heat and metabolic by-products of active tissues; stimulating the healing process; and reducing chronic edema and hematoma [54]. Therefore, vibration exercises are recommended for patients with disease requiring mucosal relaxation, those with edema, and those in the late postoperative period, in order to assist in the healing process.

On the basis of the results of the present study, we hypothesize that vocal fold vibration during trill exercises occurs as follows:

1st. The point of articulation (such as the lips and the tongue) creates oscillating occlusion of the vocal tract;

2nd. Pulmonary airflow increases in order to maintain articulatory and phonatory oscillation;

3rd. Intraoral pressure oscillates as the point of articulation vibrates, changing the phonation pressure threshold;

4th. Vocal fold vibration changes as supraglottic pressure oscillates, and we can therefore postulate that the vibratory motion of the point of articulation "massages" the vocal folds.

The Coanda effect and vortices can explain the interaction described above. Increased airflow increases the Coanda effect and the number of vortices in the glottic and supraglottic regions. It is likely that these phenomena prolong the closing phase and, consequently, increase the number of higher harmonics. However, it is likely that the vibratory motion of the point of articulation causes the vortices to have an oscillatory behavior throughout the vocal tract, the closing phase of the vocal folds varying according to the variation in pressure in that region. In the present study, we found a strong interaction between the source and the filter, as previously reported [3] [4] [22] [51] [52] [56]-[60].

Our results support the idea that vocal fold vibration occurs in a complex and nonlinear manner and can be affected by certain variables, in accordance with the principles of chaos theory [61]. If the airflow is constant, the vocal tract is free, and the vocal fold structure is in proper conditions, vocal fold vibration will be periodic. If there is a change in any of the abovementioned variables (or in any other interfering variable), periodicity will change and, depending on the extent of the change, vocal fold vibration will become completely aperiodic. In the case of trill exercises, changes are primarily due to increased airflow and filter obstruction.

We sought to study vibration in singers to try and isolate the effects of the exercise from other possible vibrational irregularities. However, we believe that, for people who present pathologies, the exercise may function differently, which is why it must be studied in many possible situations. Moreover, the intrinsic muscle movement during the exercise and the blood flow in the area must be closely analyzed so that its indication is even more informed.

\section{Conclusions}

In terms of the EGG waveform, tongue trills and lip trills are similar regardless of the intensity at which they are 
performed (i.e. high or low); however, it differs from the sustained vowel $/ \varepsilon /$ produced at high or low intensity, as follows:

- Vocal fold vibration is periodic during phonation of the sustained vowel $/ \varepsilon /$ and quasi-periodic during trill exercises;

- The oscillation pattern of the EGG waveform and the EGG signal hovers around $24 \mathrm{~Hz}$ for both trill exercises.

\section{References}

[1] Warren, D.W., Allen, G. and King, H.A. (1984) Physiologic and Perceptual Effects of Induced Anterior Open Bite. Folia Phoniatrica et Logopaedica, 36, 164-173. http://dx.doi.org/10.1159/000265736

[2] Warren, D.W., Rochet, A.P., Dalston, R.M. and, Mayo, R. (1992) Controlling Changes in Vocal Tract Resistance. Journal of the Acoustical Society of America, 91, 2947-253. http://dx.doi.org/10.1121/1.402930

[3] Titze, I.R. (2006) Voice Training and Therapy with a Semi-Occluded Vocal Tract: Rationale and Scientific Underpinnings. Journal of Speech Language and Hearing Research, 49, 448-459. http://dx.doi.org/10.1044/1092-4388(2006/035)

[4] Story, B.H., Laukkanen, A.M. and, Titze, I.R. (2000) Acoustic Impedance of an Artificially Lengthened and Constricted Vocal Tract. Journal of Voice, 14, 455-469. http://dx.doi.org/10.1016/S0892-1997(00)80003-X

[5] Gaskill, C.S. and Erickson, M.L. (2008) The Effect of a Voiced Lip Trill on Estimated Glottal Closed Quotient. Journal of Voice, 22, 634-643. http://dx.doi.org/10.1016/j.jvoice.2007.03.012

[6] McGowan, R.S. (1992) Tongue-Tip Trills and Vocal-Tract Wall Compliance. Journal of the Acoustical Society of America, 91, 2903-2910. http://dx.doi.org/10.1121/1.402927

[7] Behlau, M., Rodrigues, S., Azevedo, R. and Gonçalves MI, P.P. (1997) Avaliação e terapia de voz. In: Lopes-Filho, O., Ed., Tratado de Fonoaudiologia, Rocca, São Paulo, 607-658.

[8] Behlau, M.M.G., Feijo, D. and Pontes, P. (12001) Avaliação de Voz. In: MS, B., Ed., A voz do Especialista, 85-275.

[9] RT S (1991) Professional Voice: The Science and Art of Clinical Care. Raven Press, New York.

[10] Speyer, R. (2008) Effects of Voice Therapy: A Systematic Review. Journal of Voice, 22, 565-580. http://dx.doi.org/10.1016/j.jvoice.2006.10.005

[11] Pinho, S.M. (2001) Terapia Vocal. In: Pinho, S.M., Ed., Tópicos em Voz, Guanabara-Koogan, Rio de Janeiro.

[12] Pinho, S.M. (2003) Fundamentos em fonoaudiologia: Tratando os distúrbios da voz. Guanabara-Koogan, Rio de Janeiro.

[13] Pinho, S. and Pontes, P. (2006) Desvendando os segredos da voz: Músculos intrínsecos da laringe e dinâmica vocal. Revinter, Rio de Janeiro.

[14] Scarpel, R.D. and Pinho, S.M. (2001) Aquecimento e desaquecimento vocal. In: Pinho, S.M., Ed., Tópicos em Voz, Guanabara-Koogan, Rio de Janeiro.

[15] Maniecka-Aleksandrowicz, B., Domeracka-Kołodziej, A., Rózak-Komorowska, A. and Szeptycka-Adamus, A. (2006) Management and Therapy in Functional Aphonia: Analysis of 500 Cases. Otolaryngology Journals, 60, 191-197.

[16] Rodrigues, M. (2001) Estudo do exercício de vibração sonorizada de língua nas laringectomias frontolaterais. PUC, São Paulo.

[17] Schwarz, K. and Cielo, C.A. (2009) Vocal and Laryngeal Modifications Produced by the Sonorous Tongue Vibration Technique. Pró-Fono, 21, 161-166. http://dx.doi.org/10.1590/S0104-56872009000200013

[18] Rodrigues, S. (1995) Análise múltipla do efeito da técnica de vibração sonorizada de língua em indivíduos adultos sem queixa vocal. Universidade Federal de São Paulo, São Paulo.

[19] Bueno, T. (2006) Técnica de vibração sonorizada de língua: aspectos do aprendizado, dos efeitos acústicos e das imagens do trato vocal e da face. PUC, São Paulo.

[20] Cordeiro, G.F. (2010) Análise comparativa da amplitude de vibração das pregas vocais e do coeficiente de contato durante a emissão da vogal /ع/ prolongada e vibração sonorizada de lábios e língua. Universidade de São Paulo, São Paulo.

[21] Hamdan, A.L., Nassar, J., Al Zaghal, Z., El-Khoury, E., Bsat, M. and Tabri, D. (2012) Glottal Contact Quotient in Mediterranean Tongue Trill. Journal of Voice, 26, 669.e11-669.e15. http://dx.doi.org/10.1016/j.jvoice.2011.07.008

[22] Cordeiro, G.F., Montagnoli, A.N., Nemr, N.K., Menezes, M.H. and Tsuji, D.H. (2012) Comparative Analysis of the Closed Quotient for Lip and Tongue Trills in Relation to the Sustained Vowel /ع/. Journal of Voice, 26, e17-e22. http://dx.doi.org/10.1016/j.jvoice.2010.07.004 
[23] Moisik, S.R., Esling, J.H. and Crevier-Buchman, L. (2010) A High-Speed Laryngoscopic Investigation of Aryepiglottic Trilling. The Journal of the Acoustical Society of America, 127, 1548-1558. http://dx.doi.org/10.1121/1.3299203

[24] Vieira, M.N., McInnes, F.R. and Jack, M.A. (1997) Comparative Assessment of Electroglottographic and Acoustic Measures of Jitter in Pathological Voices. Journal of Speech Language and Hearing Research, 40, 170-182. http://dx.doi.org/10.1044/jslhr.4001.170

[25] Pinho, S.M. (2003) Fundamentos em fonoaudiologia: Tratando os distúrbios da voz. Guanabara-Koogan, Rio de Janeiro.

[26] Titze, I.R. (1988) The Physics of Small-Amplitude Oscillation of the Vocal Folds. The Journal of the Acoustical Society of America, 83, 1536-1552. http://dx.doi.org/10.1121/1.395910

[27] Fisher, K.V. and Swank, P.R. (1997) Estimating Phonation Threshold Pressure. Journal of Speech Language and Hearing Research, 40, 1122-1129. http://dx.doi.org/10.1044/jslhr.4005.1122

[28] Titze, I.R. (1992) Phonation Threshold Pressure: A Missing Link in Glottal Aerodynamics. The Journal of the Acoustical Society of America, 91, 2926-2935. http://dx.doi.org/10.1121/1.402928

[29] Jiang, J.J., Zhang, Y. and Stern, J. (2001) Modeling of Chaotic Vibrations in Symmetric Vocal Folds. The Journal of the Acoustical Society of America, 110, 2120-2128. http://dx.doi.org/10.1121/1.1395596

[30] Erath, B.D., Peterson, S.D., Zañartu, M., Wodicka, G.R. and Plesniak, M.W. (2011) A Theoretical Model of the Pressure Field Arising from Asymmetric Intraglottal Flows Applied to a Two-Mass Model of the Vocal Folds. The Journal of the Acoustical Society of America, 130, 389-403. http://dx.doi.org/10.1121/1.3586785

[31] Alipour, F. and Scherer, R.C. (2000) Vocal Fold Bulging Effects on Phonation Using a Biophysical Computer Model. Journal of Voice, 14, 470-483. http://dx.doi.org/10.1016/S0892-1997(00)80004-1

[32] Alipour, F. and Scherer, R.C. (2000) Dynamic Glottal Pressures in an Excised Hemilarynx Model. Journal of Voice, 14, 443-454. http://dx.doi.org/10.1016/S0892-1997(00)80002-8

[33] Alipour, F., Finnegan, E.M. and Scherer, R.C. (2009) Aerodynamic and Acoustic Effects of Abrupt Frequency Changes in Excised Larynges. Journal of Speech Language and Hearing Research, 52, 465-481. http://dx.doi.org/10.1044/1092-4388(2008/07-0212)

[34] Tokuda, I.T., Horacek, J., Svec, J.G. and Herzel, H. (2007) Comparison of Biomechanical Modeling of Register Transitions and Voice Instabilities with Excised Larynx Experiments. The Journal of the Acoustical Society of America, 122, 519-531. http://dx.doi.org/10.1121/1.2741210

[35] Nomura, H. and Funada, T. (2006) Numerical Simulation of Unsteady Flow through the Rigid Glottis. Acoustical Science and Technology, 27, 154-162. http://dx.doi.org/10.1250/ast.27.154

[36] Tao, C., Zhang, Y., Hottinger, D.G. and Jiang, J.J. (2007) Asymmetric Airflow and Vibration Induced by the Coanda Effect in a Symmetric Model of the Vocal Folds. The Journal of the Acoustical Society of America, 122, 2270-2278. http://dx.doi.org/10.1121/1.2773960

[37] Becker, S., Kniesburges, S., Muller, S., Delgado, A., Link, G., Kaltenbacher, M., et al. (2009) Flow-Structure-Acoustic Interaction in a Human Voice Model. The Journal of the Acoustical Society of America, 125, 1351-1361. http://dx.doi.org/10.1121/1.3068444

[38] Khosla, S., Murugappan, S. and Gutmark, E. (2008) What Can Vortices Tell Us about Vocal Fold Vibration and Voice Production. Current Opinion in Otolaryngology \& Head and Neck Surgery, 16, 183-187. http://dx.doi.org/10.1097/MOO.0b013e3282ff5fc5

[39] Triep, M. and Brücker, C. (2010) Three-Dimensional Nature of the Glottal Jet. The Journal of the Acoustical Society of America, 127, 1537-1547. http://dx.doi.org/10.1121/1.3299202

[40] Kirmse, C., Triep, M., Brücker, C., Döllinger, M. and Stingl, M. (2010) Experimental Flow Study of Modeled Regular and Irregular Glottal Closure Types. Logopedics Phoniatrics Vocology, 35, 45-50. http://dx.doi.org/10.3109/14015431003667652

[41] Zheng, X., Mittal, R., Xue, Q. and Bielamowicz, S. (2011) Direct-Numerical Simulation of the Glottal Jet and Vocal-Fold Dynamics in a Three-Dimensional Laryngeal Model. The Journal of the Acoustical Society of America, 130, 404-415. http://dx.doi.org/10.1121/1.3592216

[42] Zheng, X., Mittal, R. and Bielamowicz, S. (2011) A Computational Study of Asymmetric Glottal Jet Deflection during Phonation. The Journal of the Acoustical Society of America, 129, 2133-2143. http://dx.doi.org/10.1121/1.3544490

[43] Okuno, E., Caldas, I.L. and Chow, C. (1982) Física para ciências biológicas e biomédicas. Editora Harbra, São Paulo.

[44] Hofmans, G.C., Groot, G., Ranucci, M., Graziani, G. and Hirschberg, A. (2003) Unsteady Flow through in Vitro Models of the Glottis. The Journal of the Acoustical Society of America, 113, 1658-1675. http://dx.doi.org/10.1121/1.1547459

[45] Oliveira, P.M. (2008) Sustentação aerodinâmica: O mecanismo físico [Internet]. 
http://dited.bn.pt/31619/2606/3166.pdf

[46] Alipour, F. and Scherer, R.C. (2004) Flow Separation in a Computational Oscillating Vocal Fold Model. The Journal of the Acoustical Society of America, 116, 1710-1719. http://dx.doi.org/10.1121/1.1779274

[47] Sciamarella, D. and Quere, P.L. (2008) Solving for Unsteady Airflow in a Glottal Model with Immersed Moving Boundaries. European Journal of Mechanics B/Fluids, 128, 42-53. http://dx.doi.org/10.1016/j.euromechflu.2007.06.004

[48] Mihaescu, M., Khosla, S.M., Murugappan, S. and Gutmark, E.J. (2010) Unsteady Laryngeal Airflow Simulations of the Intra-Glottal Vortical Structures. The Journal of the Acoustical Society of America, 127, 435-444. http://dx.doi.org/10.1121/1.3271276

[49] Dejonckere, P.H. (1986) Acoustic Analysis of Voice Production. Production Trial from a Clinical Perspective. Acta Oto-Rhino-Laryngologica Belgica, 40, 377-385.

[50] Haji, T., Horiguchi, S., Baer, T. and Gould, W.J. (1986) Frequency and Amplitude Perturbation Analysis of Electroglottograph during Sustained Phonation. The Journal of the Acoustical Society of America, 80, 58-62. http://dx.doi.org/10.1121/1.394083

[51] Titze, I.R. (2008) Nonlinear Source-Filter Coupling in Phonation: Theory. The Journal of the Acoustical Society of America, 123, 2733-2749. http://dx.doi.org/10.1121/1.2832337

[52] Titze, I., Riede, T. and Popolo, P. (2008) Nonlinear Source-Filter Coupling in Phonation: Vocal Exercises. The Journal of the Acoustical Society of America, 123, 1902-1915. http://dx.doi.org/10.1121/1.2832339

[53] Pinho, S.M. (2001) Terapia Vocal. In: Pinho, S.M., Ed., Tópicos em Voz, Guanabara-Koogan, Rio de Janeiro, 1-18.

[54] Delisa, J.A. (1992) Medicina de reabilitação: Princípios e prática. Manole, São Paulo.

[55] Macardle, W.D., Kach, F.I. and Katch, V.L. (1985) Fisiologia do exercício: Energia, nutrição e desempenho humano. Guanabara, Rio de Janeiro.

[56] Titze, I.R. and Story, B.H. (1997) Acoustic Interactions of the Voice Source with the Lower Vocal Tract. The Journal of the Acoustical Society of America, 101, 2234-2243. http://dx.doi.org/10.1121/1.418246

[57] Titze, I.R. and Laukkanen, A.M. (2007) Can Vocal Economy in Phonation Be Increased with an Artificially Lengthened Vocal Tract? A Computer Modeling Study. Logopedics Phoniatrics Vocology, 32, 147-156. http://dx.doi.org/10.1080/14015430701439765

[58] Laukkanen, A.M., Titze, I.R., Hoffman, H. and Finnegan, E. (2008) Effects of a Semioccluded Vocal Tract on Laryngeal Muscle Activity and Glottal Adduction in a Single Female Subject. Folia Phoniatrica et Logopaedica, 60, 298311. http://dx.doi.org/10.1159/000170080

[59] Titze, I.R. (2009) Phonation Threshold Pressure Measurement with a Semi-Occluded Vocal Tract. Journal of Speech Language and Hearing Research, 52, 1062-1072. http://dx.doi.org/10.1044/1092-4388(2009/08-0110)

[60] Menezes, M.H., Ubrig-Zancanella, M.T., Cunha, M.G., Cordeiro, G.F., Nemr, K. and Tsuji, D.H. (2011) The Relationship between Tongue Trill Performance Duration and Vocal Changes in Dysphonic Women. Journal of Voice, 25, e167-e175. http://dx.doi.org/10.1016/j.jvoice.2010.03.009

[61] Hirsch, M.W., Samale, S. and Devaney, R.L. (2004) Differential Equations, Dynamical Systems-An Introduction to Chaos. 2nd Edition, Elsevier Academic Press, New York. 